\title{
Estresse e Autoconceito em Pais e Mães de Crianças com a Síndrome do X-Frágil
}

\section{Stress and Self-concept in Parents of Children with Fragile X-Syndrome}

\author{
Zuleika Ana Cherubini*, Cleonice Alves Bosa \& Denise Ruschel Bandeira \\ Universidade Federal do Rio Grande do Sul, Porto Alegre, Brasil
}

\begin{abstract}
Resumo
A síndrome do x-frágil é a principal causa de deficiência mental herdada, suplantada apenas pela síndrome de Down, sendo a mulher a principal transmissora. Indivíduos com a síndrome do X-frágil apresentam um conjunto de características que pode causar um impacto no sistema familiar. Este estudo investiga o estresse e o autoconceito em 15 pais e mães de meninos com a síndrome do X-frágil, 15 de meninos com Síndrome de Down e 15 de meninos com desenvolvimento típico, baseado no modelo biopsicossocial de Bradford. Os resultados revelaram uma diferença significativa no autoconceito (self pessoal) entre as mães de crianças com a Síndrome do X-frágil e as mães de crianças com desenvolvimento típico, mas não entre aquelas e o grupo $\mathrm{S}$. de Down, sugerindo que o fator da transmissão por si só não explica as diferenças no autoconceito. Não houve diferença entre os grupos na medida de estresse.

Palavras-chave: Síndrome do X-Frágil; Síndrome de Down; estresse parental; autoconceito; apoio social.
\end{abstract}

\begin{abstract}
The Fragile X Syndrome is the major inherited intellectual disability, superseded only by Down Syndrome, and it is transmitted mostly by females. Patients with Fragile X Syndrome show a set of physical, clinical, behavioral and cognitive features that may impact the family structures, especially the parental figures. This study investigates the stress and self-concept of 15 parents of boys with Fragile X Syndrome, 15 parents of boys with Down syndrome and 15 with typical development accordingly to the biopsychosocial model of Bradford. The results showed a significant difference of self-concept between mothers of children with Fragile X Syndrome and typical developmental groups, but not in those with Down Syndrome, suggesting that the transmission factor solely does not explain differences in self-concept.

Keywords: Fragile X Syndrome; Down Syndrome; parental stress; self-concept; social support.
\end{abstract}

Nos últimos anos, a síndrome do X-Frágil (SXF) tem sido tema de investigação freqüente pela área médica (Watson, 2005) por suas peculiaridades genéticas e moleculares (O’Donell \& Warren, 2002). Poucos estudos têm sido realizados na área psicológica para investigar o impacto da presença de um indivíduo portador desta síndrome na família.

Vários estudos têm sido desenvolvidos sobre o estresse desencadeado pela presença de um membro da família com uma doença crônica (Bauer et al., 2000; Hamlett, Pellegrini, \& Katz, 1992; Ong, Afifah, Sofiah, \& Lye, 1998) ou com alguma deficiência física ou mental (Milgran \& Atzil, 1988; Pereira-Silva \& Dessen, 2001; Perry, McGarvey, \& Factor, 1992; Ryde-Brandt, 1990). Contudo, são poucas as pesquisas que associam o estresse parental às características de um familiar com a SXF (Johnston et al., 2003; Von Gontard et al., 2002).

*Endereço para correspondência: Consultório Privado de Psicologia, Ramiro Barcelos, 1793/408, Rio Branco, Porto Alegre, RS, 90035-006. E-mails: zcherubini@gmail.com, cleobosa@uol.com.bredrbandei@terra.com.br
A SXF é geneticamente transmitida e é reconhecida como a primeira causa de deficiência mental herdada (Hoogeveen \& Oostra, 1997; Plomin, DeFries, McClearn, \& Rutter, 1997; Turner, Webb, Wake, \& Robinson, 1996). Apresenta vários sinais e sintomas clínicos, físicos, comportamentais e cognitivos, tais como: macroorquidismo, macrocefalia, palato ogival alto, queixo e orelhas proeminentes, problemas oftalmológicos, cardíacos, otites, dificuldades motoras, fono-articulatórias, viso-espaciais, de raciocínio aritmético, déficit na atenção, deficiência mental, agressividade, hiperatividade e autismo, entre outras (Hagerman, 2002).

O sofrimento representado pelo estresse nos pais de uma criança com a SXF é evidenciado antes mesmo do diagnóstico, quando o comportamento do filho(a) sugere algo incomum. Bailey (2003) revelou, em suas pesquisas, que a busca pelo diagnóstico é um dos fatores mais relevantes de estresse físico e emocional.

De forma geral, estudos têm demonstrado que as figuras parentais são as mais afetadas pelo estresse em casos de desenvolvimento atípico de um filho, sendo, porém, a mãe mais atingida por sua condição de cuidadora direta (Hamlett, et al., 1992; Ong et al., 1998). Apesar dos meca- 
nismos do impacto do estresse na saúde ainda serem elusivos, o excesso de tarefas atribuídas a uma pessoa gera conseqüências em sua saúde física (Bauer et al., 2000; Epel et al., 2004) e psicológica (Bandeira et al., 2007; Bandeira, Gonçalves, \& Pawlowski, 2006). A saúde psi-cológica dos cuidadores tem sido tema de investigação, porque a função de "cuidar" foi identificada como um fator de estresse crônico (Lazarus \& DeLongis, 1983). Para autores como Carrion, Córdoba e Collado (2003) e Milgran e Atzil (1988), as mulheres são mais propensas a serem influenciadas pelos aspectos afetivos e pessoais da vida, ao passo que os homens o são pelas condições econômicas e profissionais.

Ainda em relação às diferenças de gênero, Bristol, Gallagher e Schopler (1988) investigaram o impacto dos transtornos do desenvolvimento na adaptação e nos papéis parentais (pai e mãe), utilizando pais de filhos com desenvolvimento típico como controle. Os autores chamaram a atenção para a relação diretamente proporcional entre o bem-estar psicológico e o suporte conjugal. Os achados demonstraram que, em ambos os grupos, as mães revelaram mais sintomas depressivos, sugerindo que outros fatores, além da doença, podem gerar estresse. Em relação à divisão de tarefas, os pais de crianças sem TGD assumiram igualmente a responsabilidade e os cuidados dos filhos; já as mães de crianças com TGD revelaram sentimentos de maior sobrecarga, e os pais, de menor responsividade às demandas impostas pela doença. Além desses indicadores, tanto os pais como as mães da criança afetada sinalizaram algum conflito conjugal e a percepção de várias interferências na vida diária, entretanto as mães relataram mais problemas do que os pais. O estudo concluiu que, em ambos os casos, as mães relacionaram o bem-estar psicológico ao apoio do marido.

Outros estudos têm chegado a conclusões semelhantes, como por exemplo, a recente pesquisa de Schmidt (2004). Este autor investigou 30 mães de crianças autistas e encontrou altos níveis de estresse, porém sem prejuízos em sua auto-eficácia. Os resultados apontaram que a qualidade do apoio social e as estratégias de coping foram mediadores no efeito de estresse materno e nos sentimentos de autoeficácia.

De acordo com Hessl et al. (2001), a sintomatologia presente na criança com a SXF está associada aos transtornos afetivos presentes nos pais. Para esses autores, as características de comportamento de uma criança com essa síndrome afetam o status psicológico dos pais, interferindo na qualidade da interação com sua criança. Johnston et al. (2003) investigaram os principais fatores associados ao estresse em mães de crianças afetadas pela SXF. Os resultados de tal investigação confirmaram que os problemas de comportamento presentes na criança com a SXF são determinantes para o desenvolvimento do estresse na mãe, destacando o comprometimento na fala, o déficit de atenção, as complicações somáticas, a agressividade e o comprometimento social como as características comportamentais mais desencadeadoras de estresse. Além do fator comportamental, concluiu-se que a qualidade da coesão familiar, que engloba o compartilhamento de tarefas e responsabilidades, assim como o apoio familiar estão relacionados ao nível de estresse na mãe. Nesses casos, quando as mães recebem apoio e assistência de outros membros da família frente aos cuidados da criança, elas tendem a desenvolver sentimentos de maior confiança em relação ao seu desempenho e à sua função materna, diminuindo os de solidão e estresse.

Contrário a muitos estudos que correlacionam o estresse dos pais à deficiência mental do filho(a) (Dyson, 1997; Perry et al., 1992), Johnston et al. (2003) não encontraram associação significativa entre essas duas variáveis, sugerindo que o estresse parental está mais diretamente relacionado à dificuldade de manejar o comportamento de uma criança com a SXF do que aos déficits cognitivos nela presentes. Os autores ressaltam que o diagnóstico definitivo e o esclarecimento das características da síndrome contribuem para aliviar o estresse nos pais, já que estes podem desenvolver expectativas mais realistas sobre o futuro.

Diante de um quadro como a SXF, cuja transmissão se dá entre as gerações, sendo a mãe, em sua maioria, a principal transmissora, é possível que, além dos sentimentos de estresse presentes, possa existir algum prejuízo no autoconceito parental. Segundo Tamayo (1981), o autoconceito pode ser definido como um conjunto de autopercepções, como por exemplo, traços, sentimentos e imagens, as quais são passíveis de mudanças durante a interação com o outro. Este constructo é composto pelas dimensões somática (self somático: percepções do seu próprio corpo somadas às percepções do outro); pessoal (self pessoal: características psicológicas que atribui a si); social (self social: características de interação com o outro); ético-moral (self ético-moral: imagem de dignidade moral).

A recente pesquisa de McConkie-Rosell, Spiridigliozzi, Sullivan, Dawson e Lachiewicz, (2000) buscou explorar a configuração do autoconceito em mulheres sob risco de herança da SXF. A avaliação foi realizada antes e depois do teste genético para a SXF, tendo como variáveis o autoconceito global pela TSCS (Tennessee Self-concept) e os sentimentos sobre si mesma. Os resultados apontaram ausência de diferenças significativas entre portadores e nãoportadores da SXF no autoconceito global. Entretanto, após o teste genético, as não-portadoras referiram aumento dos sentimentos positivos sobre si mesmas, enquanto o oposto ocorreu com as portadoras. Cinco áreas específicas foram identificadas: implicações de um teste positivo para seu próprio filho, possível barreira para ter filhos biológicos, expressão das características clínicas em si mesma, alteração de sua identidade genética e culpa por não saber de sua condição. Nesse estudo, o self-físico não apresentou diferenças, apesar da consciência das portadoras sobre suas características clínicas. No self-social não houve diferenças aparentes relacionadas ao status de risco ou de portador. Quanto à identidade genética, esse estudo chamou a atenção para o relato de algumas mulheres, comparando o efeito do mecanismo de transmissão ao de uma "bomba genética". Assim, essa pesquisa indicou leves alterações na identidade relacionada à possibilidade de ser portadora da SXF. 
Em termos de gênero, Carrion, Córdoba e Collado (2003) verificaram que as mulheres são mais sensíveis e responsáveis diante da necessidade de outras pessoas e se envolvem emocionalmente mais que os homens frente aos acontecimentos da vida. Segundo eles, essa tendência pode ser explicada em função das diferenças de autoconceito de cada sexo, as quais podem estar associadas às influências sociais que determinam a forma de pensar, agir e comportar-se. Neste sentido, é concebível que as características de gênero estão implicadas num contexto social e são observadas através dos comportamentos masculino e feminino, assim como o contexto social interfere na formação do autoconceito.

O impacto da SXF na família, em especial no pai e na mãe, encobre uma série de fatores de ordem individual e social, já que se trata de um transtorno do desenvolvimento que sugere a necessidade de enfoques multidisciplinares. A revisão sobre o impacto de uma doença na família e o seu respectivo ajustamento foi bem sistematizada por Bradford (1997) na área da doença crônica. Trata-se de um metamodelo biopsicossocial que integra a abordagem sistêmica e cognitiva e que pode ser utilizado na compreensão dos fenômenos presentes nos transtornos do desenvolvimento, em especial devido ao fator crônico. Sendo assim, o autor chama a atenção para a forma como a família lida com os estressores decorrentes da doença e para as condições dos sistemas presentes e interatuantes no loccus da doença, tais como: o padrão de interação familiar, a comunicação médico-paciente-família, as estratégias de coping, as crenças, o sistema de saúde, o apoio social, os desafios específicos da doença e as características individuais. $\mathrm{O}$ autor também chama a atenção para o fato de que a ausência de doença não sugere saúde familiar, mas a forma como a família maneja e se adapta às demandas de uma patologia. Assim, a adaptação familiar depende das características do estressor, moderado pelos recursos disponíveis e pelas crenças familiares.

O modelo biopsicossocial é uma abordagem abrangente, uma vez que busca associar os aspectos da doença em si, o comportamento do paciente, o contexto social e familiar no qual está inserido, bem como o sistema de saúde (Pereira, 2002). Sabe-se que a doença é um grande mobilizador do estresse no indivíduo cuidador, porém pode ser "aliviado" diante de vários fatores, tais como apoio conjugal ou social, crenças, padrão de comunicação e estratégias de coping.

Este estudo investiga o estresse e o autoconceito em pais e mães de meninos com a SXF, SD e desenvolvimento típico (DT), baseado no modelo biopsicossocial de Bradford (1997). As hipóteses são que pais e mães dos grupos SXF e SD apresentam níveis mais elevados de estresse do que os do grupo DT; que as mães do grupo SXF apresentam maior nível de estresse e maior prejuízo em seu autoconceito do que os pais desse mesmo grupo, considerando serem as mães as transmissoras; e que as mães do grupo SXF apresentam um autoconceito negativo se comparado às mães dos grupos SD e DT.

\section{Método}

Foi utilizado um delineamento de Grupos Contrastantes (Naschmias \& Naschmias, 1996) a fim de avaliar as diferenças do nível de estresse e o autoconceito entre os grupos de pais e mães de meninos diagnosticados com a SXF, pais e mães de meninos com a síndrome de Down e pais e mães de meninos com desenvolvimento típico.

\section{Participantes}

Noventa participantes, representados por 15 pais e mães de meninos diagnosticados por estudo molecular com a SXF; 15 pais e mães de meninos com Síndrome de Down (SD) decorrente da trissomia livre e 15 pais e mães de meninos com desenvolvimento típico (DT). Os integrantes do grupo SXF foram recrutados pelo serviço de genética do Hospital de Clínicas de Porto Alegre (HCPA) e da Fundação Brasileira da SXF. Os do grupo SD em escolas especiais e na Associação de Familiares e Amigos de Down (AFAD) e os do grupo DT em escolas de ensino comum. Os critérios de inclusão consistiram em serem pais biológicos nos três grupos, residirem com o filho e não apresentarem doença física que impedisse seu cuidado. Como critérios de exclusão foram considerados a presença de Deficiência Mental no Teste Matrizes Progressivas de Raven em mães do grupo SXF e os casos de Síndrome de Down não decorrentes da trissomia livre.

A escolha pelo gênero masculino entre os filhos dos casais participantes do estudo é explicada pela maior predisposição destes para desenvolver a mutação completa e, conseqüentemente, com o fenótipo determinado para a SXF. A opção pela síndrome de Down como um grupo-controle se deu pela necessidade de controlar os aspectos relativos ao atraso de desenvolvimento global, bem como por sua condição não-transgeracional.

A média de idades das mulheres no grupo SXF foi 36,40 anos $(D P=7,99)$ e dos homens de 39,47 anos $(D P=8,85)$. Nas mulheres do grupo $\mathrm{SD}$, a média foi de 40,07 anos $(D P$ $=7,33)$ e nos homens de 44,67 anos $(D P=6,29)$. No grupo DT, as mulheres tinham 39,47 anos em média $(D P=4,56)$ e os homens 43,53 $(D P=4,81)$. A média de idade dos portadores da SXF foi de 11,80 anos $(D P=5,25)$, dos da SD foi de 8,80 anos $(D P=4,28)$ e nos de desenvolvimento típico foi de 8,40 anos $(D P=2,95)$.

Quanto à escolaridade, há predomínio do ensino médio nos dois grupos clínicos (SXF e SD), salientando-se que apenas $26,7 \%$ das mães SXF ingressaram em curso superior. O grupo de mães DT, por sua vez, configura o maior percentual no ensino superior $(46,7 \%)$ em comparação aos grupos clínicos. A porcentagem de pais que freqüentam ou freqüentaram nível superior é maior do que a das mães nos grupos clínicos. Em relação ao fator profissional, percebese que $73,4 \%$ das mães SXF trabalham, sendo que $26,7 \%$ exercem atividades sem vínculo empregatício (doméstica, artesã e costureira) e 26,7\% têm carga horária flexível de até 4 horas diárias. Diferentemente delas, todos os pais deste grupo trabalham, com carga horária de 5 a 8 horas diárias, sendo que a categoria ocupacional que apresentou 
maior concentração $(26,7 \%)$ foi relativa às atividades comerciais (vendas e prestações de serviços). No grupo SD, as mães que trabalham $(73,4 \%)$ atuam, predominantemente, na área administrativa (20\%) e em serviços técnicos (20\%). Essas mães trabalham até 4 horas $(20,0 \%)$ e de 5 a 8 horas $(20,0 \%)$. Todos os pais trabalham (100\%), sendo que um terço deles $(33,3 \%)$ exerce serviços diversos sem vínculo empregatício (eletricista, auxiliar de obras, serviços gerais), ocupando uma jornada de trabalho de 5 e 8 horas diárias $(46,7 \%)$. No grupo DT, a maioria das mães também trabalha (73\%), porém em torno de 5 a 8 horas diárias e com cargos gerenciais e públicos. Apenas um pai desse grupo não trabalha, e 26,7\% exercem cargos gerenciais. Interessante notar que as mães dos três grupos apresentam percentuais semelhantes em termos de distribuição de categoria profissional, porém a diferença recai sobre a jornada de trabalho diária, uma vez que as mães SXF possuem menor disponibilidade para o trabalho.

\section{Instrumentos e Procedimentos}

A Ficha de dados sociodemográficos objetivou caracterizar os participantes, colher dados clínicos e identificar possíveis estressores, além de obter informações relacionadas às crianças com a SXF e SD (tratamentos, características, presença ou ausência de apoio e orientação, tipos de crença em relação à doença).

O Mini International Neuropsychiatric Interview Brasilian version 5.0.0 - MINI (Sheehan et al., 1998) é uma entrevista diagnóstica padronizada breve, compatível com os critérios do DSM-II-R/IV e da CID-10, destinada à determinação dos principais eixos de distúrbios psiquiátricos. A entrevista foi administrada individualmente pela pesquisadora responsável, com duração média de 15 a 20 minutos. Esse instrumento foi validado para o Brasil (Amorin, 2000).

O Inventário de Sintomas de Estresse para Adultos de Lipp - ISSL (Lipp, 2000), destinado a medir a sintomatologia de estresse (física ou psicológica), bem como identificar a fase de estresse (alerta, resistência, quase exaustão e exaustão) no indivíduo, é composto de 37 itens de natureza somática e 19 de natureza psicológica de auto-relato. Essa escala obteve um ótimo nível de consistência interna ( $\alpha=$ $0,93)$, similar ao obtido pela autora $(\alpha=0,91)$.

A Escala Fatorial de Autoconceito - EFA (Tamayo, 1981), que foi empregada para avaliar a variável dependente Autoconceito, tem validade fatorial e avalia as dimensões dos selves somático, pessoal, social e ético-moral. Este instrumento é composto por 51 itens de atributos bipolares de uma escala de 7 pontos, sendo que a maior pontuação num dos fatores corresponde à dimensão hierárquica determinante do autoconceito do indivíduo. Neste estudo, a EFA obteve um ótimo nível de consistência interna $(\alpha=0,89)$, próximo ao estudo do autor do instrumento $(\alpha=0,95)$.

Por último, o Teste de Matrizes Progressivas de Raven (Raven, 2003) é um teste não-verbal utilizado como medida de inteligência com base no referencial teórico de Spearman e validado para a realidade brasileira. Em forma de caderno com 60 problemas, foi respondido pelas mães do grupo SXF, a fim de detectar deficiência mental. Nenhuma delas atingiu um escore que indicasse deficiência mental. Sua fidedignidade oscila entre 0,83 e 0,93 , segundo a idade.

Após a assinatura do termo de consentimento livre e esclarecido, foi agendada uma visita à residência do casal para a administração dos instrumentos.

\section{Resultados}

De acordo com o MINI, nas mães do grupo SXF, a maior incidência de distúrbios psiquiátricos foi de agorafobia (26,7\%), seguida de transtorno de pânico $(20,0 \%)$. Já no grupo de mães $\mathrm{SD}$, houve maior incidência de ansiedade generalizada $(40,0 \%)$ e depressão $(53,3 \%)$, ressaltando-se o risco de suicídio (13,3\%). A depressão também aparece no grupo de mães DT $(26,7 \%)$, porém com menor incidência. Nos pais, episódios maníacos ou hipomaníacos foram encontrados nos três grupos, em especial no grupo SD. Entretanto, os pais do grupo SXF são os que apresentam menores percentuais de sintomas psiquiátricos, quando comparados às mães e aos pais dos outros grupos.

Sobre o estresse, os resultados do ISSL são melhor visualizados na Tabela 1. A análise de Qui-quadrado mostra que não há diferença significativa entre os grupos em relação à presença de estresse; no entanto pais e mães do grupo DT revelaram menor ocorrência de estresse, quando comparados aos grupos clínicos. Quanto às fases de estresse, apenas a da resistência é identificada em todos os grupos, com predomínio nas mães do grupo SXF (53,3\%). Diante da presença de estresse, sintomas físicos e psicológicos foram identificados em mães do grupo SXF na mesma proporção $(26,7 \%)$. Nota-se que, nos pais desse grupo, não há presença de sintomas físicos relacionados ao estresse, mas sim, psicológicos $(40,0 \%)$. Da mesma forma, verifica-se a ausência de sintomatologia de estresse físico nas mães de crianças com desenvolvimento típico. Não houve correlação dos resultados do ISSL e a idade.

Finalmente, sobre as peculiaridades de cada diagnóstico (SXF e SD), a Tabela 2 apresenta as maiores dificuldades percebidas pelos pais e pelas mães em relação ao seu filho. No caso do grupo SXF, as mães percebem o comprometimento na área social (isolamento, dificuldades na interação social) como o mais problemático $(73,3 \%)$. Além da área social, pais e mães do grupo SXF também relataram a presença de estereotipias, problemas comportamentais (hiperatividade, agressividade, irritabilidade), de atenção (concentração) e de ansiedade em seus filhos. Por outro lado, indicações de estereotipias, dificuldades na atenção (concentração) e ansiedade não são apontados pelos pais e mães do grupo SD.

Diante da condição do filho, as freqüências das percepções dos pais em relação às variáveis Apoio e Orientação mostraram que as mães tendem a sentir menos apoio do que os pais nos dois grupos. No entanto, chama a atenção que, para ambos os grupos, especialmente as mães SXF $(53,8 \%)$, o tipo de apoio recebido está voltado para os cui- 
Cherubini, Z. A., Bosa, C. A. \& Bandeira, D. R. (2008). Estresse e Autoconceito em Pais e Mães de Crianças com a Síndrome do X-Frágil

Tabela 1

Freqüências e Percentuais do Diagnóstico de Estresse, Fase e Tipo de Sintomatologia por Grupo e Sexo (n=60)

\begin{tabular}{|c|c|c|c|c|c|c|}
\hline \multirow[b]{2}{*}{ Estresse $f(\%)$} & \multicolumn{2}{|c|}{$S X F$} & \multicolumn{2}{|l|}{$S D$} & $D T$ & \\
\hline & Pai & Mãe & Pai & Mãe & Pai & Mãe \\
\hline Sem estresse & $9(60,0)$ & $7(46,7)$ & $13(86,7)$ & $8(53,3)$ & $12(80,0)$ & $12(80,0)$ \\
\hline \multicolumn{7}{|l|}{ Fase do Estresse } \\
\hline Alerta & - & - & - & - & - & - \\
\hline Resistência & $6(40,0)$ & $8(53,3)$ & $2(13,3)$ & $7(46,7)$ & $3(20,0)$ & $3(20,0)$ \\
\hline Exaustão & - & - & - & - & - & - \\
\hline Quase Exaustão & - & - & - & - & - & - \\
\hline \multicolumn{7}{|l|}{ Sintomatologia } \\
\hline Psicológico & $6(40,0)$ & $4(26,7)$ & $1(6,7)$ & $4(26,7)$ & $2(13,3)$ & $3(20,0)$ \\
\hline Físico & - & $4(26,7)$ & $1(6,7)$ & $3(20,0)$ & $1(6,7)$ & - \\
\hline
\end{tabular}

Tabela 2

Freqüências e Percentuais das Dificuldades do Filho Percebidas pelos Pais por Grupo e Sexo $(n=60)$

\begin{tabular}{llrrr}
\hline & \multicolumn{3}{c}{$S X F$} & \multicolumn{2}{c}{$S D$} \\
\cline { 2 - 5 } Dificuldades $f(\%)$ & \multicolumn{1}{c}{ Pai } & \multicolumn{1}{c}{ Mãe } & \multicolumn{1}{c}{ Pai } & \multicolumn{1}{c}{ Mãe } \\
\hline Relac. Social & $8(53,3)$ & $11(73,3)$ & $4(26,7)$ & $7(46,7)$ \\
Estereotipias & $4(26,7)$ & $4(26,7)$ & $0(0,0)$ & $0(0,0)$ \\
Linguagem & $2(13,3)$ & $2(13,3)$ & $6(40,0)$ & $7(46,7)$ \\
Agitação & $3(20,0)$ & $4(26,7)$ & $2(13,3)$ & $1(6,7)$ \\
Comportamento & $4(26,7)$ & $4(26,7)$ & $4(26,7)$ & $8(53,3)$ \\
Aprendizagem & $4(26.7)$ & $2(13,3)$ & $2(13,3)$ & $4(26,7)$ \\
Concentração & $2(13,3)$ & $2(13,3)$ & $0(0,0)$ & $0(0,0)$ \\
Ansiedade & $2(13,3)$ & $3(20,0)$ & $0(0,0)$ & $0(0,0)$ \\
Saúde & $0(0,0)$ & $1(6,7)$ & $1(6,7)$ & $0(0,0)$ \\
\hline
\end{tabular}

dados da criança. Quanto aos pais, a percepção de apoio no grupo SXF é reconhecida na troca de experiências e no diálogo (33,3\%), diferentemente dos pais do grupo SD que o identifica nos cuidados $(54,5 \%)$. Em ambos os grupos e gêneros, $o$ apoio advém do cônjuge e dos familiares.

Sobre a existência ou não de orientação, as mães dos meninos com a SXF relatam receber pouca orientação, quando comparadas aos pais e ao grupo SD. Para essas mães, o tipo de orientação recebida está voltado, predominantemente, para o manejo do filho $(30,8 \%)$. Novamente, nos dois grupos, os pais e as mães reconhecem os profissionais como a maior fonte de orientação, assim como fundações e associações, ainda que em menor escala.

Na Tabela 3, é possível verificar a associação entre estresse e orientação recebida $\left(\chi^{2}=10,36\right.$ a $\left.p<0,002\right)$ onde o percentual maior de ausência de estresse ocorreu no grupo de pais e mães que recebem orientação sobre as respectivas síndromes. Para ambos os grupos, o maior percentual de orientação recebida advém dos profissionais e é dirigida, em sua maior parte, para as questões de manejo do filho.

As subescalas da EFA foram analisadas através de Análise de Variância Univariada, com os fatores grupo e sexo.
Tabela 3

Freqüências e Percentuais entre as Variáveis Presença de Orientação e Niveis de Estresse nos Grupos Clínicos

\begin{tabular}{lcc}
\hline$f(\%)$ & Sem Estresse & Estresse de Resistência \\
\hline Sem Orientação & $4(26,7)$ & $11(73,3)$ \\
Com Orientação & $31(73,8)$ & $11(26,2)$ \\
\hline
\end{tabular}

Nota. $\left(\chi^{2}=10,36\right.$ a $\left.p<0,002\right)$.

A Tabela 4 apresenta os resultados das médias em cada subescala. Os resultados na subescala do self-somático mostraram que não há diferença significativa para grupos e sexo nem interação de fatores. Para a subescala self-pessoal, os resultados apontaram interação entre grupo e sexo $[F(2,87)=5,137 ; p=0,008]$. Analisando-se os intervalos de confiança, pode-se perceber que a diferença se encontra entre as mães do grupo SXF e as mães do grupo DT (4,53 $-5,35$ e 5,63-6,46), significando que existe uma percepção negativa de si mesma nas mães de crianças com a SXF, se comparada às do grupo DT. Quanto ao self-social, não 
foram encontradas diferenças significativas entre grupos e sexo, nem interação de fatores. Na subescala self-éticomoral há diferença entre os sexos. Analisando-se os grupos separadamente, através do teste $t$, observou-se que essa diferença se encontra no grupo SD ( $t=2,22, g l=28 ; p=$ $0,034)$. Isso significa que, neste grupo, os pais se percebem menos éticos moralmente do que as mães.

Tabela 4

Médias e Desvios-Padrão das Subescalas do Autoconceito por Grupo e Sexo

\begin{tabular}{lcccccc}
\hline & \multicolumn{2}{c}{ SXF } & \multicolumn{2}{c}{ SD } & & DT \\
\cline { 2 - 7 } Autoconceito $M(D P)$ & Pai & Mãe & Pai & Mãe & Pai & Mãe \\
\hline Self Somático & $4,79(0,98)$ & $5,03(0,73)$ & $5,04(0,80)$ & $4,60(1,21)$ & $5,27(1,02)$ & $5,25(0,93)$ \\
Self Pessoal & $5,67(0,73)$ & $4,93(0,83)^{\mathrm{a}}$ & $5,47(0,78)$ & $5,76(0,60)$ & $5,53(1,22)$ & $6,04(0,35)^{\mathrm{a}}$ \\
Self Social & $5,08(0,69)$ & $5,33(0,45)$ & $4,98(0,77)$ & $5,11(0,53)$ & $5,12(0,93)$ & $5,50(0,57)$ \\
Self Ético-Moral & $6,45(0,32)$ & $6,55(0,51)$ & $6,39(0,53)^{\mathrm{b}}$ & $6,73(0,26)^{\mathrm{b}}$ & $6,55(0,53)$ & $6,65(0,34)$ \\
\hline
\end{tabular}

Nota. Letras iguais apontam diferenças : a) $[F(2,87)=5,137$ e $p=0,008]$; b) $[t(28)=2,22$ e $p=0,03]$.

\section{Discussão}

De modo geral, os resultados do MINI demonstraram a ocorrência de ansiedade e fobias nas mães do grupo SXF. Esses resultados corroboram os reportados na literatura (Franke et al., 1998; Freund, Reiss, Hagerman, \& Vinogradov, 1992; Reiss, Hagerman, Vinogradov, Abrams, \& King, 1988). Entretanto, não é possível estabelecer uma relação causal, uma vez que é difícil explicar se esses resultados relacionam-se à condição de pré-mutação das mães do grupo SXF ou à de cuidadora, já que no grupo SD também houve ocorrência de ansiedade.

Sobre o estresse, os resultados não revelaram diferença significativa entre pais e mães do grupo SXF e, tampouco entre os três grupos investigados. Houve muitas semelhanças entre os grupos clínicos, sugerindo que o fator herança genética talvez não seja um marcador significativo na avaliação do estresse. Isso pode ter ocorrido em função do tamanho pequeno da amostra ou de outros fatores que possam ter amenizado o estresse, tal como a existência de suporte familiar e profissional nos grupos clínicos. De fato, as mães dos grupos clínicos referem ter obtido apoio de seus maridos e familiares nos cuidados de seus filhos.

Quanto às fases do estresse, identificou-se a de resistência, sendo que as freqüências foram muito semelhantes nos grupos de pais de filhos com transtorno de desenvolvimento (SXF e SD). Essa fase, segundo Lipp (2000), representa uma tentativa do organismo de reagir e resistir a um acúmulo de estressores. De fato, este estudo mostrou que, para as mães do grupo SXF, as características do comportamento (oposicionismo, hiperatividade), as dificuldades na área social (isolamento, dificuldade de interação, fobias, preconceito social) e as estereotipias são consideradas de difícil manejo, exigindo, portanto, maior disponibilidade de atenção e cuidado. As mães do grupo SD também reconheceram a necessidade de maior dedicação ao seu filho pelas dificuldades comportamentais (teimosia, inquietação), pelos distúrbios na linguagem e na área social. Tais limitações também implicam tratamentos especializados à criança, o que mobiliza tempo e disponibilidade por parte do cuidador. Esses achados são semelhantes às conclusões da pesquisa de Johnston (2003), ao identificarem que as características de comportamento da criança com SXF foram determinantes no estresse físico e psicológico das mães, sendo mais associado às dificuldades de manejo comportamental do filho do que aos déficits cognitivos nele encontrados.

Apesar de ser um indicador de excesso de demandas, a fase de Resistência ainda não é considerada patológica, garantindo ao indivíduo condições de dar conta das situações de vida. Recentemente, Poehlmann, Clements, Abbeduto e Farsad (2005) demonstraram a importância do contexto, das reações emocionais, do uso de estratégias de coping e da adaptação familiar como elementos que interferem na construção de sentimentos de disrupção ou resiliência mediante o reconhecimento do diagnóstico. Para PereiraSilva e Dessen (2001), os sentimentos dos pais de uma criança com deficiência mental são determinantes para a adaptação e o bem-estar familiar. Por exemplo, culpa, mágoa e frustrações reforçam o estresse e interferem negativamente na adaptação e interação familiar a esta condição. Portanto, o grau de adaptação aos problemas crônicos de saúde e os recursos de coping utilizados para lidar com adversidades geradas por estas dependem da percepção subjetiva do cuidador (Beresford, 1994), do tipo de incapacitação, suas interfaces (prognóstico, dor, tratamento, sintomas, curso) e do grau de vulnerabilidade e resistência (Bradford, 1997). Nesse sentido, o modelo sistêmico considera as variáveis saúde, coping, suporte, crenças, padrões de interação, desafios da doença e características individuais como fatores de um sistema que interferem positiva ou negativamente na forma como a família lida com os estressores. A presença de apoio social, incluindo, nesse conceito, a orientação, assim como as crenças adotadas são 
fatores importantes e necessários para aliviar o impacto da doença em um sistema familiar.

Para Beresford (1994), o apoio conjugal é um dos mais significativos recursos de coping e está relacionado a resultados positivos na condução da doença, assim como o apoio de familiares alivia o estresse físico e emocional do cuidador. Os resultados, nesse estudo, mostraram que as mães dos dois grupos clínicos sentem menos apoio do que os pais; entretanto reconhecem que existe um apoio advindo de seus parceiros e de suas famílias, nas questões de cuidado e afeto. Diferentemente das mães, poucos pais de cada grupo reconheceram falta de apoio, talvez por sentirem que as mães sustentam com maior propriedade as carências e assumem mais as dificuldades inerentes à pressão da condição da criança. Conforme Bradford (1997), diante de um fator mobilizador é comum a família sentir-se vulnerável ao desenvolvimento de sintomas de estresse e, por isso, a presença de apoio emocional de outros funciona como um fator de proteção.

A importância da relação médico-família-paciente, considerada na abordagem sistêmica como um dos fatores interatuantes no processo de bem-estar familiar, foi corroborada neste estudo, na medida em que os profissionais foram considerados como fonte de apoio e identificados como aqueles que melhor orientam sobre as características das síndromes. Através deles, a família parece sentir-se amparada diante da angústia e das dúvidas acerca do diagnóstico, o que aponta a necessidade de outros estudos para investigar mais detalhadamente esta questão. A maioria das mães, dos dois grupos, sinalizou que a principal orientação recebida é relativa ao manejo comportamental por seus sentimentos de insegurança, diante da condição da criança. Como são as mães, em geral, que mais lidam diretamente com a criança, são elas que ficam mais suscetíveis a dúvidas sobre sua competência materna, necessitando, talvez, de maior orientação. De fato, este estudo mostrou haver uma associação entre a presença de estresse e orientação recebida.

Sobre a informação do diagnóstico, o estudo de Souza, Nista, Scotono e Guerreiro (1998) mostrou que tanto os pais como as mães se sentem mais capacitados para lidar com seu filho, após serem informados sobre o diagnóstico. Entretanto, no estudo de Bristol et al. (1988) e no de Johnston et al. (2003), a adaptação materna e o grau de impacto do diagnóstico dependeram especialmente do apoio do pai e das crenças sobre o mesmo, e não da severidade da SXF. Mais especificamente, o nível de estresse dependeu mais das dificuldades de manejo do que das limitações cognitivas do filho. Sendo assim, a presença de apoio social e familiar parece elevar a confiança dos pais em relação ao próprio desempenho e amenizar os sentimentos de solidão e estresse. Dyson (1997) concorda com esta premissa ao concluir que o apoio possibilita maior bem-estar e alivia os sentimentos de sobrecarga e estresse.

Com relação ao autoconceito, os resultados revelaram diferença significativa no self pessoal entre mães dos grupos SXF e DT, mas não entre as mães SXF e SD. Nesse sentido, é interessante reconhecer que nem o self somático que é representado pela auto-imagem corporal, nem o self social que significa a percepção sobre a capacidade de interação com os outros, distinguiram as mães destes dois grupos. McConkie-Rosell et al. (2000) ao avaliarem o autoconceito em mães portadoras e não-portadoras da SXF, também não encontraram diferenças nas dimensões do self físico (somático) e social, mas sim no self pessoal das mães portadoras. Sendo assim, o status genético pode ser um marcador para uma percepção negativa de si mesmo, mas não pode explicar por si só tais resultados. Por isso, é importante refinar esses achados através de outras medidas para investigar os sentimentos da mãe enquanto transmissora da SXF ao seu filho.

A diferença estatisticamente significativa encontrada na dimensão self pessoal mostrou que as mães SXF percebem-se mais inseguras para conduzir suas vidas e com dúvidas sobre sua competência, corroborando os achados de Souza et al. (1998) quando mostraram que mães de crianças epilépticas são mais inseguras e intranqüilas do que os pais. Aqui cabe comentar as questões de gênero no autoconceito reportadas por Carrion, et al. (2003). Para eles, as características femininas e masculinas estão implicadas num contexto social e determinam a forma de pensar, agir e comportar-se.

A questão da transmissão da SXF pelas mães sugere um impacto na percepção de si mesma devido aos sentimentos desencadeados por essa condição. Assim como Bailey, Skinner, Hatton e Roberts (2000) reconheceram uma percepção negativa de si mesmas nas mulheres portadoras da SXF, pode-se entender que os resultados aqui encontrados sobre o self pessoal nas mães dos grupos clínicos podem se relacionar a sentimentos de culpa e de dúvidas quanto a sua capacidade de "parentalizar" uma criança com problemas de desenvolvimento, os quais podem ou não estar atrelados à condição da transmissão genética da síndrome. Assim como o nível de estresse está vinculado às condições de outros fatores biopsicossociais, o mesmo pode acontecer com o autoconceito, uma vez que sua construção está diretamente relacionada a aspectos interpessoais, isto é, à percepção individual adquirida através do círculo social (Nurius, 1986). Neste sentido, Bosa, Cavalheiro, Vignochi e Mittman (2003) também chamaram a atenção para o impacto do estigma na saúde mental familiar, ao reconhecer que as reações sociais podem superdimensionar uma dada sintomatologia e, portanto, repercutir na auto-estima.

Sabe-se que o trabalho fora de casa também exerce um papel importante na saúde física e mental de um indivíduo e favorece a construção de uma rede social. A impossibilidade de exercer uma ocupação que promova um desenvolvimento e satisfação também pode ser um dos fatores desencadeantes de um autoconceito negativo. Pode-se entender que as mães de crianças com desenvolvimento típico não revelaram um autoconceito negativo não só pela ausência de doença na família, mas também, possivelmente, por disporem de oportunidades de investimento pessoal e profissional. Em função disso, é possível inferir que o 
status ocupacional possa ter influenciado na construção de um autoconceito mais prejudicado nas mães SXF. Para Einam e Cuskelly (2002), o trabalho exerce um papel protetivo às mães, pois pode agir como um redutor de uma auto-avaliação negativa.

De forma geral, este estudo mostrou a interferência de fatores biopsicossociais no nível de estresse e no autoconceito dos pais diante do impacto da doença do filho. Em especial, foi significativo reconhecer que pais e mães apresentam algum tipo de estresse diante das dificuldades do filho, assim como mostrou que a condição de transmissão genética não parece ser o principal marcador para as poucas diferenças encontradas. É importante que as dimensões de estresse e o perfil do autoconceito sejam identificados, compreendidos e considerados para que se consiga amenizar o impacto negativo deste tipo de diagnóstico na vida dos pacientes e de suas famílias. O autoconceito tem um papel importante para o bem-estar do indivíduo, por ser um importante regulador e mediador do comportamento, das percepções e expectativas pessoais, sendo uma importante dimensão a ser considerada no atendimento à família.

Apesar de vários estudos apontarem maior estresse e problemas de autoconceito em famílias de crianças com algum transtorno de desenvolvimento, isso não ocorreu no presente estudo, assim como não se confirmou a noção de que estes aspectos seriam influenciados pela transmissão genética. Várias condições podem ter contribuído para este resultado como, por exemplo, o tamanho reduzido da amostra e possíveis dificuldades na seleção dos grupos comparativos, em especial o de SD. De fato, chamou a atenção, neste estudo, a necessidade de maior apoio a este último grupo. Neste sentido, a ocorrência de uma rede de apoio no grupo com SXF foi justamente um dos fatores que pode ter contribuído para a ausência de níveis mais severos de estresse.

\section{Referência}

Amorin, P. (2000). Mini International Neuropsychiatric Interview (MINI): Validação de entrevista breve para diagnóstico de transtornos mentais. Revista Brasileira de Psiquiatria, 22, 106-15.

Bailey, D. B. (2003). Discovering Fragile X syndrome: Family experiences and perceptions. Pediatrics, 111, 407- 416.

Bailey, D. B., Skinner, D., Hatton, D., \& Roberts, J. (2000). Family experiences and factors associated with the diagnosis of Fragile X syndrome. Developmental and Behavioral Pediatrics, 21, 315-321.

Bandeira, D. R., Bozzetti, M. C., Gonçalves, T. R., Hilgert, J. B., Hugo, F. N., \& Pawlowski, J. (2007). Psychological distress in brasilian caregivers of relatives with dementia. Aging and Mental Health, 11, 14-19.

Bandeira, D. R., Gonçalves, T. R., \& Pawlowski, J. (2006). Envelhecimento e dependência: Impacto sobre familiarescuidadores de portadores de síndrome demencial. In M. A. M. P. Parente (Ed.), Cognição e envelhecimento (pp. 275-284). Porto Alegre, RS: ArtMed.
Bauer, M. E, Vedhara, K., Perks, P., Wilcock, G. K., Lightman, S. L., \& Shanks, N. (2000). Chronic stress in caregivers of dementia patients is associated with reduced lymphocyte sensitivity glucocorticoids. Journal of Neuroimmunology, 103, 84-92.

Beresford, B. A. (1994). Resources and strategies: How parents cope with the care of a disabled child. Journal of Child Psychology and Psychiatry, 35, 171-209.

Bosa, C. A, Cavalheiro, E. P, Vignochi, L., \& Mittman, N. (2003). Experiências de portadores de X-frágil em grupos de escoteiros: A criação de espaços de socialização. Projeto de Extensão. Manuscrito não publicado.

Bradford, R. (1997). Children, families and chronic disease: Psychological models and methods of care. London: Routledge.

Bristol, M., Gallagher, J., \& Schopler, E. (1988). Mothers and fathers of young developmentally disabled and nondisabled boys: Adaptation and spousal support. Developmental Psychology, 24, 441-451.

Carrion, A. C., Córdoba, A. I., \& Collado, A. G. (2003). Diferencias en la percepción de influencia de los acontecimientos vitales en hombres y mujeres. Revista Latinoamericana de Psicología, 35, 19-26.

Dyson, L. L. (1997). Fathers and mothers of school-age children with developmental disabilities: Parent stress, family functioning, and social support. American Journal on Mental Retardation, 102, 267-279.

Einam, M., \& Cuskelly, M. (2002). Paid employment of mothers and fathers of an adult child with multiple disabilities. Journal of Intellctual Disabilitie Research, 46, 158-167.

Epel, E. S., Blackburn, E. H., Lin, J., Dhabhar, F. S., Adler, N. E., Morrow, J. D., et al. (2004). Accelerated telomere shortening in response to life stress. Proceedings of the Rational Academic of Sciences USA, 101, 17312-17315.

Franke, P., Leboyer, M., Gansicke, M., Weiffenback, O., Biancalana, V., Cornillet-Lefebre, P., et al. (1998). Genotypephenotype relationship in female carriers of the permutation and full mutation of FMR-1. Psychiatric Research, 80, 113-27.

Freund, L. S., Reiss, A. L., Hagerman, R., \& Vinogradov, S. (1992). Chromosome fragility and psychopatology in obligate female carriers of the Fragile X syndrome. Archives of Genetic Psychiatry, 49, 54-60.

Hagerman, R. J. (2002). The Fragile X syndrome: Diagnosis, treatment, and research. Baltimore, MD: The Johns Hopkins University Press.

Hamlett, K. W., Pellegrini, D. S., \& Katz, K. S. (1992). Childhood chronic ilness as a family stressor. Journal of Pediatric Psychology, 17, 33-47.

Hessl, D., Dyer-Friedman, J., Glaser, B., Wisbeck, J., Barajas, G. R., Taylor, A., et al. (2001). The influence of environmental and genetic factors on behavior problems and autistic symptoms in boys and girls with Fragile X syndrome. Pediatrics, 108, 76-88.

Hoogeveen, A. T., \& Oostra, B. A. (1997). The Fragile X syndrome. Journal of Inherit Metabolism Disorder, 20, 139-151.

Johnston, C., Hessl, D., Blasey, C., Eliez, S., Erba, H., DyerFriedman, J., et al. (2003). Factors associated with parenting stress in mothers of children with Fragile $\mathrm{X}$ syndrome. Developmental and Behavioral Pediatrics, 4, 267-275. 
Lazarus R. S., \& DeLongis, A. (1983). Psychological stress and coping in aging. American Psychologist, 38, 245-254.

Lipp, M. (2000). Manual do Inventário de Sintomas de Stress para Adultos de Lipp (ISSL). São Paulo, SP: Casa do Psicólogo.

McConkie-Rosell, A., Spiridigliozzi, G. A., Sullivan, J. A., Dawson, D. V., \& Lachiewicz, A. M. (2000). Carrier testing in Fragile X syndrome: Effect on self-concept. American Journal of Medical Genetics, 92, 336-342.

Milgran, N. A., \& Atzil, M. (1988). Parenting stress in raising autistic children. Journal of Autism and Developmental Disorders, 18, 415-424.

Naschmias, C., \& Naschmias, D. (1996). Research methods in the Social Sciences. London: Arnold.

Nurius, P. S. (1986). Reappraisal of the self-concept and implications for counseling. Journal of Counseling Psychology, 33, 429-438.

O’Donell, W. T., \& Warren, S. T. (2002). A decade of molecular studies of Fragile X syndrome. Annual Review of Neuroscience, 25, 315-338.

Ong, L. C., Afifah, I., Sofiah, A., \& Lye, M. S. (1998). Parenting stress among mothers of Malaysian children with cerebral palsy: Predictors of child-and parent-related stress. Annals of Tropical Pediatrics, 18, 301-307.

Pereira, M. G. (2002). A perspectiva biopsicossocial na avaliação em Psicologia da saúde: Modelo interdependente. Psicologia: Teoria, Investigação e Prática, 2, 185-193.

Pereira-Silva, N. L., \& Dessen, M. A. (2001). Deficiência mental e família: Implicações para o desenvolvimento da criança. Psicologia: Teoria e Pesquisa, 17, 133-141.

Perry, A., McGarvey, N., \& Factor, D. C. (1992). Stress and family functioning in parents of girls with Rett syndrome. Journal of Autism and Developmental Disorders, 22, 235-248.

Plomin, R., DeFries, J. C., McClearn, G. E., \& Rutter, M. (1997). Behavioral genetics. New York: W. H. Freeman.

Poehlmann, J., Clements, M., Abbeduto, L., \& Farsad, V. (2005). Family experiences associated with a child's diagnosis of Fragile X or Down syndrome: Evidence for disruption and resilience [Eletronic version]. Mental Retardation, 43, 255267.

Raven, J. C. (2003). Manual do teste Matrizes Progressivas Escala Geral (F. Campos, Trad. \& Adapt.). Rio de Janeiro, RJ: Cepa.

Reiss, A. L., Hagerman, R. J., Vinogradov, S., Abrams, M., \& King, R. J. (1998). Psychiatric disability in female carriers of the Fragile X chromosome. Archives of Genetic Psychiatry, 45, 25-30.

Ryde-Brandt, B. (1990). Anxiety and depression in mothers of children with psychotic disorders and mental retardation. British Journal of Psychiatry, 156, 118-121.

Schmidt, C. (2004). Estresse, auto-eficácia e o contexto de adaptação familiar de mães de portadores de transtornos globais do desenvolvimento. Dissertação de Mestrado não-publicada, Curso de Pós-Graduação em Psicologia do Desenvolvimento, Universidade Federal do Rio Grande do Sul, Porto Alegre, RS. Sheehan, D. V., Lecrubier, Y., Harnett-Sheehan, K., Amorim, P., Janavs, J., Weiller, E., Hergueta, T., Baker, R., \& Dunbar, G. (1998). The Mini International Neuropsychiatric Interview (MINI): The development and validation of a structured diagnostic psychiatric interview. Journal of Clinical Psychiatry, 59, 22-33.
Souza, E. P., Nista, C. R., Scotono, A. E., \& Guerreio, M. M (1998). Sentimentos e reações de pais de crianças epilépticas. Arquivos de Neuropsiquiatria, 56, 39-44. Retrieved July, 15, 2005, from htpp//www.scielo.br/php

Tamayo, A. (1981). Escala Fatorial de Autoconceito. Arquivos Brasileiros de Psicologia, 33, 87-102.

Turner, G., Webb, T., Wake, S., \& Robinson, H. (1996). Prevalence of Fragile X syndrome. American Journal of Medical Genetics, 88, 11-24.

Von Gontard, A., Backes, M., Laufersweiler-Plass, C., Wendland, C., Lehmkuhl, G., Zerres, K., et al. (2002). Psychopathology and familial stress-comparison of boys with Fragile X syndrome and spinal muscular atrophy. Journal of Child Psychology and Psychiatry, 43, 949-957.

Watson, J. D. (2005). DNA: O segredo da vida (C. A. Malferrair, Trad.). São Paulo, SP: Companhia das Letras.
Recebido: 25/01/2007 $1^{a}$ revisão: $27 / 09 / 2007$ Aceite final: 05/12/2007 